\title{
A code biology analysis of the regulatory regions in cell lines
}

\section{Análisis de biología de códigos de las regiones reguladoras en líneas celulares}

\author{
Omar Paredes?', Isaías May-Canche', Elena Fimmel² \\ 'Universidad de Guadalajara \\ ${ }^{2}$ Mannheim University of Applied Sciences
}

\begin{abstract}
Coding sequences are widely studied for their relevance in protein synthesis. However, higher organism genomes, such as human genomes, has a small amount of them, and a larger proportion of non-coding sequences. ENCODE and Epigenomic Roadmap projects discovered that regulatory functions are carried out in the non-coding regions of the human genome. These regulatory functions are part of the regulatory machinery that yields different gene expression profiles, thus, different cell lines. Whereas different environmental elements, i. e. histone modifications, DNA methylation, and other epigenomic phenomena, determine the regulatory function of genome part, the sequences' composition where these functions take place could also influence regulatory machinery. In this work, we explore the non-coding regulatory sequences and lexica build with subsequences between 3 and 16 nucleotides to evaluate the difference between the sequence composition of the regulatory regions in the cell lines. Our results show that the lexica corresponding to the regulatory regions are different based on their complexity/degeneracy, moreover, the lexica of regulatory regions in different cell lines are also different. These features suggest that non-coding sequences are an active element of the regulatory machinery and the histone code that are involved in cell differentiation.
\end{abstract}




\section{RESUMEN}

Las secuencias codificantes han sido ampliamente estudiadas por su relevancia en la síntesis de proteínas. Sin embargo, los genomas de organismos complejos, como el humano, tiene una porción menor de estas secuencias y una mayor proporción de secuencias no codificantes. Los proyectos del ENCODE y Epigenomic Roadmap describieron que las funciones reguladoras se llevan acabo en las regiones no codificantes del genoma humano. Estas funciones reguladoras son parte de la maquinaria reguladora que produce diferentes perfiles de expresión genética, por tanto, diferentes líneas celulares. Mientras diferentes elementos del entorno, como las modificaciones en las histonas, metilación del ADN y otros fenómenos epigenéticos, determinan la función reguladora que tienen una porción del genoma, la composición de la secuencia donde estas funciones son llevadas a cabo también podrían influir en la maquinaria reguladora. En este trabajo, se exploraron las secuencias de las regiones no codificantes y los léxicos generados con las subsecuencias entre 3 y 16 nucleótidos, para evaluar las diferencias entre la composición de las secuencias de las regiones reguladoras en las líneas celulares. Los resultados muestran que los léxicos correspondientes a las regiones reguladoras son diferentes con base en su complejidad/degeneración, así mismo, los léxicos de las regiones reguladoras en distintas líneas celulares son también distintos. Estos detalles sugieren que las secuencias no codificantes son elemento activo de la maquinaria reguladora y del código histónico que participan en la diferenciación celular.

Palabras Clave: Complejidad de Léxico; Regiones Reguladoras; Biología de Códigos

\section{Correspondencia}

DESTINATARIO: Omar Paredes

INSTITUCIÓN: Univerisdad de Guadalajara

DIRECCIÓN: Blvd. Gral. Marcelino García Barragán \#1421,

C. P. 44430, Guadalajara, Jalisco, México

CORREO ELECTRÓNICO: omar.paredes@alumnos.udg.mx

\section{Fecha de recepción:}

21 de septiembre de 2018

Fecha de aceptación:

11 de enero de 2019 


\section{INTRODUCTION}

DNA sequences are carriers of hereditary material in all living organisms ${ }^{[1]}$. The hereditary information in DNA is stored as a code made up of four chemical bases, adenine $(\mathrm{A})$, guanine $(\mathrm{G})$, cytosine $(\mathrm{C})$, and thymine $(\mathrm{T})$, written in triletter words (codons) without delimiters that are decoded after copying into a complementary RNA (transcription) into a matching protein sequence in a process called translation. In the 2000s, the Human Genome Project estimated that only approximately $2 \%$ of human genome consists of coding sequences and the remaining large part of the DNA (non-coding regions) does not serve as a template for protein sequences ${ }^{[2]}$.

However, ENCODE and Epigenomic Roadmap consortiums evidenced that there are regulatory functions in the apparently non-functional sequence of the human genome ${ }^{[3,4]}$. Both consortiums located the regulatory regions in 127 cell lines based on epigenomic profiles [4, 5], and thus, they implemented an experimental whole-genome validation of the histone code.

The histone code is a set of rules that maps the histone modifications to chromatin packaging events and leads to regulatory functions in gene expression ${ }^{[6-8]}$. Altogether, these events build a regulatory machinery that depends on the environmental context, shows diverse gene expression profiles and, hence, a diversity of cell lines ${ }^{[9,10]}$.

Elements of the previously mentioned context, that possibly determine a cell line, are locations where the chromatin packaging events happen ${ }^{[11-15]}$.

A way for studying such phenomena is suggested within an emergent discipline, Code Biology, which considers life events, for instance, as maps between organic signs and organic meanings ${ }^{[16-18]}$; in this work represented by genomic sequence and regulatory function, respectively. The Code Biology approach includes a methodology for identifying organic codes consisting of three steps: (i) demonstrating the existence of two sets linked by an organic code; (ii) identifying the decoder of the organic code, called adaptor; and (iii) validating an arbitrary nature of the organic code (compare, for instance, Hofmeyr ${ }^{[19]}$ ).

In particular, in ${ }^{[20]}$, the histone code was examined from this viewpoint: "we try to show how simple combinations of essential elements such as histone modifications can participate in sophisticated cellular features such as the structure of the genome. Here code is identified, where an input system (histone modifications) is translated into an output system (chromatin states) via adaptors (epigenetic regulators or transcription factors). Such a code has distinct importance in gene regulation and consequently for the cellular phenotype".

In this exploratory work, we implement genomic signal processing and natural language techniques to explore the sequences of regulatory regions and evidence that indeed these sequences play an important role in the regulatory machinery.

\section{METHODS}

In this work, the regulatory regions of three types of human cell lines are being explored to identify differences between the regulatory machineries in these cell lines at the sequence level. A workflow of the methodology in this work shown in Figure 1.

In order to perform this preliminary analysis, we choose the cell lines: $\mathrm{H} 1$ cells, Primary T CD8+ naive cells and Brain hippocampus middle, that represent pluripotent cells, first culture, and differentiated cells.

We download the files of the three cells corresponding to 14 regulatory regions (Table 1 ) proposed by the Epigenomic Roadmap Map project from the database of the mentioned project (http://www.roadmapepigenomics.org/data/, August 2018). 


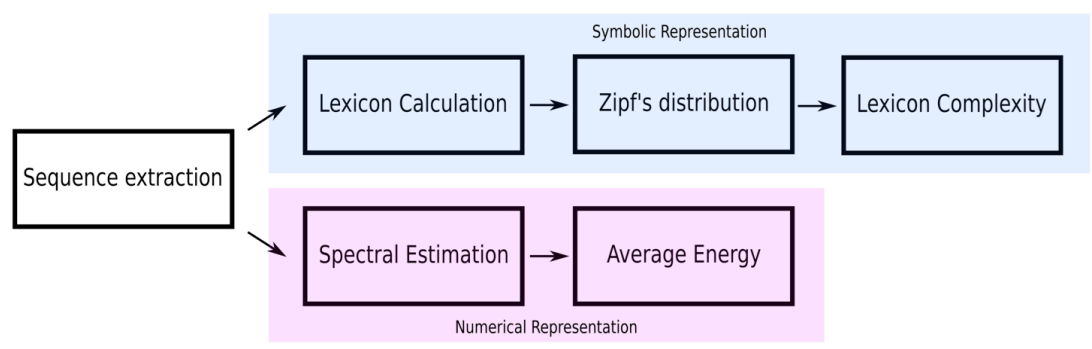

FIGURE 1. Methodology workflow.

TABLE 1. Regulatory regions proposed by the Epigenomic Roadmap Consortium ${ }^{[4]}$.

\begin{tabular}{|c|c|}
\hline \multicolumn{2}{|c|}{ Regulatory regions } \\
\hline Abbreviation & Name \\
\hline TssA & Active TSS \\
\hline TssAFlnk & Flanking active TSS \\
\hline TxFlnk & Transc. at gene 5'and 3' \\
\hline Tx & Strong transcription \\
\hline TxWk & Weak transcription \\
\hline EnhG & Genic enhancers \\
\hline Enh & Enhancer \\
\hline ZNF/Rpts & ZNF genes + repeats \\
\hline Het & Heterochromatin \\
\hline TssBiv & Bivalent/poised TSS \\
\hline BivFlnk & Flanking bivalent TSS/Enh \\
\hline EnhBiv & Bivalent enhancer \\
\hline ReprPC & Repressed Polycomb \\
\hline ReprPCWk & Weak repressed Polycomb \\
\hline
\end{tabular}

The downloaded files contain the location indices of the regulatory region in the human genome. Based on the indices, we extracted the corresponding sequences and mapped them into a genomic signal by the Voss method. In this work, we keep both representations of the DNA, sequences and the genomic signals.

The Voss method is a tetradimensional graphic of the DNA sequences that represent in each dimension a nucleotide and value the presence $x[n]=1$ and absence $x[n]=0$ of the respectively nucleotide. For example, the genomic signal of the sequence "GTCAGTCGTAA" is:

$$
\begin{aligned}
& A=[00010000011], C=[00100010000], \\
& G=[10001001000], T=[01000100100] .
\end{aligned}
$$

\section{Symbolic representation}

We classify sequences into 14 groups, where each group contains the sequences with one of the regulatory functions from Table 1 . As asserted in the Introduction, a DNA sequence can be symbolically represented as a chain of four letters (A, T, C, and G). In this representation, a word of length $k$ or k-mer is an arbitrary subsequence that contain $k$ consecutive nucleotides. It is easy to see that the number of words in a sequence of length $l$ is equal to $l-k+1$. Hereinafter, we will call these words the k-lexicon of the sequence.

According to this approach, we calculate the k-lexica for each sequence for the $k$ values from 3 to 16 nucleotide towards to identify relevant lexica in the non-coding regulatory sequences. Then we calculate the relative frequency for each word in each of the k-lexica to obtain the probability distribution of the lexicon and order the frequencies in the descending order.

The obtained distribution is the so-called Zipf's law distribution (Figure 2). The Zipf's law is a power law that describes many types of data studied in the physical and social sciences, among them the language ${ }^{[21]}$, and states, for instance, that the frequency of any word is inversely proportional to its rank in the frequency table. In the specific case of the language, the Zipf's law is a measure of the complexity/degeneracy of the language and an expression of the least effort principle of the vocabulary ${ }^{[22,23]}$. We will adopt the equation of the Zipf's law distribution in the following form: 


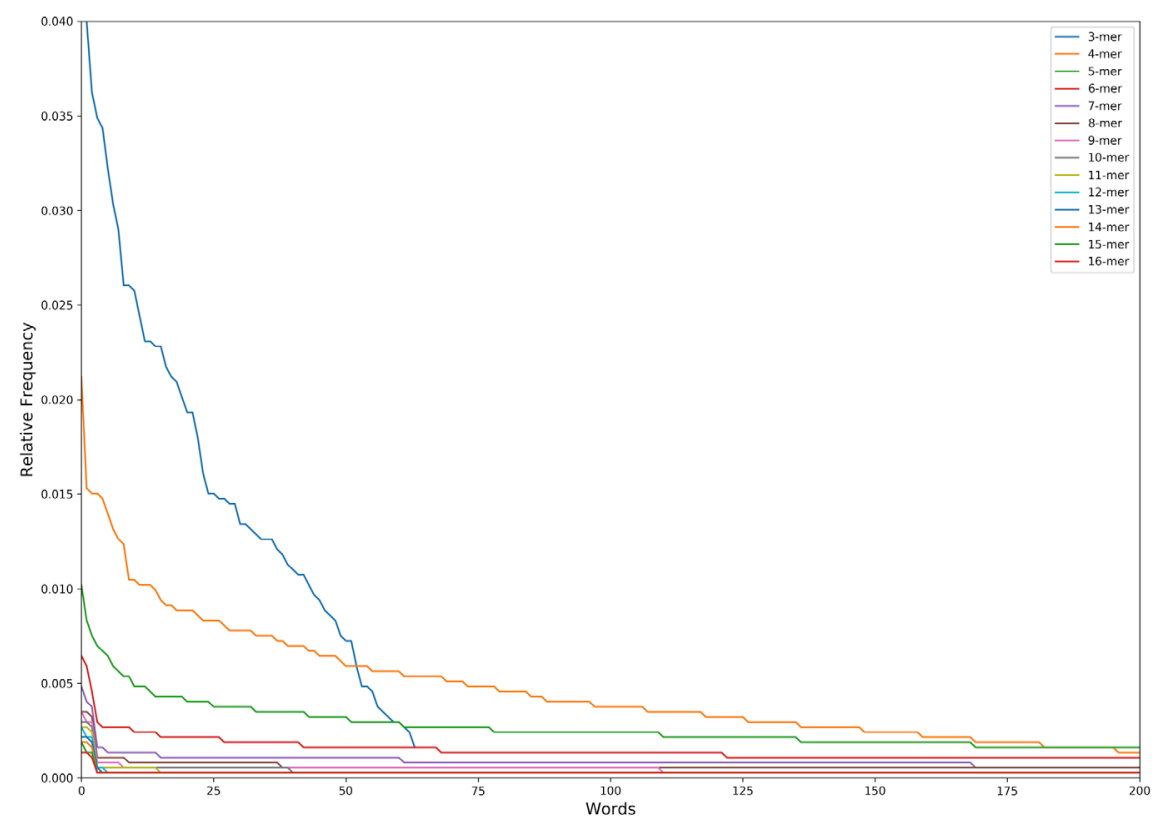

FIGURE 2. The Zipf's Law distribution of the first $\mathbf{2 0 0}$ words (except $\mathbf{3}$ nucleotides that has a maximum of 64 words lexicon) in the n-lexicon from 3 nucleotides to 16 nucleotides.

$$
g(w)=\frac{A}{r(w)^{b}}
$$

where $w$ is a word in the lexicon; $r(w)$ the absolute frequency of the word; $A$ a constant; $b$ the value of the exponent characterizing the Zipf's distribution. In this work we call $b$ the lexicon complexity, and mean the higher the value of $b$ the higher the complexity/degeneracy of the vocabulary ${ }^{[24]}$. After all, $g(w)$ denotes the relative frequency of the word $w$.

Thereafter, we linearize the Zipf's distribution by dividing each value with its respective inverse. The result distribution is now a distribution with linear behavior, which slope is the lexicon complexity. We do a linear regression by the least square method to calculate the lexicon complexity of each sequence for its vocabularies from 3 nucleotides to 16 nucleotides.

\section{Numerical representation}

For each genomic signal, we calculate its periodogram. A periodogram is a technique to obtain the frequency spectrum of a signal, in this case, a genomic signal. This technique enhances the spectrum and fixes it to a certain length, that is important to this work because of the variable lengths in the sequences of this work. We fix all the periodogram to the length of 500 values.

The equation to calculate the periodogram is given by Eq. 2 where $X[n]$ represents the periodogram of the genomic signal, $N$ the number of points, $x[n]$ is the genomic signal, in this work the Voss representation, and $f$ the frequency. An example of a periodogram is shown in Figure 3.

$$
\hat{X}[n]=\frac{1}{N} \sum_{n=0}^{N-1} \tilde{x}[n] e^{-i 2 \pi f n}
$$

After all, we divide the periodogram into frequency bands that correspond to periodicities in the genomic signal, recalling that the inverse of the frequency is the periodicity. We have then 14 intervals of frequencies (Table 2) that correspond to the same values of length, from $k=3$ to $k=16$, that we calculate for the lexicon complexity. 


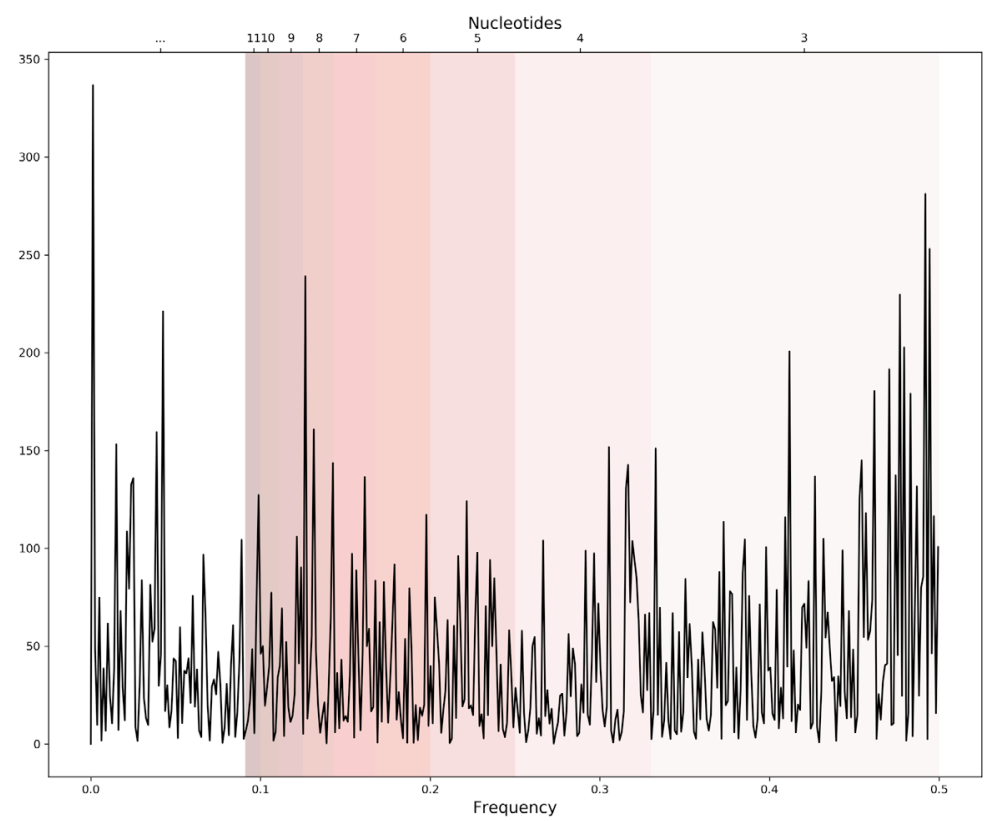

FIGURE 3. An example of a periodogram of one genomic signal. Bands of frequencies represent the pattern information of a length in the sequence correspondent to certain nucleotides i.e. 0.33-049 frequencies represent the patterns of 3 nucleotides length.

TABLE 2. Intervals of frequency and their corresponding periodicities.

\begin{tabular}{|c|c|}
\hline Periodicity (nt) & Interval of frequencies $(\mathbf{H z})$ \\
\hline 1 & $0.330-0.500$ \\
\hline 2 & $0.250-0.330$ \\
\hline 3 & $0.200-0.250$ \\
\hline 4 & $0.167-0.200$ \\
\hline 5 & $0.143-0.167$ \\
\hline 6 & $0.125-0.143$ \\
\hline 7 & $0.111-0.125$ \\
\hline 8 & $0.010-0.111$ \\
\hline 9 & $0.091-0.010$ \\
\hline 10 & $0.083-0.091$ \\
\hline 11 & $0.077-0.083$ \\
\hline 12 & $0.071-0.077$ \\
\hline 13 & $0.067-0.071$ \\
\hline 14 & $0.062-0.067$ \\
\hline 15 & $0.067-0.071$ \\
\hline 16 & $0.062-0.067$ \\
\hline
\end{tabular}

Then, we calculate the average energy of each interval to evaluate the average potential capacity to encode information in the evaluated pattern length ${ }^{[25]}$, adopting the following average energy equation

$$
E_{k}=\frac{1}{N_{k}} \sum_{n=f_{o}}^{f_{u}} \hat{X}[n]
$$

where $E_{k}$ is the energy ascribed to the periodicity o pattern length $k ; f_{o}$ is the initial frequency of the interval; $f_{u}$ the upper frequency of the interval; $X[n]$ is the periodogram of the genomic signal; and $N_{k}$ the number of points in the periodogram corresponding to pattern length $k$ frequencies.

\section{RESULTS AND DISCUSSION}

The 14 regulatory regions used in this work correspond to 1'009,178 sequences, distributed as follows: 363,513 from cell line H1 cells; 249,377 from cell line Primary T CD8 ${ }^{+}$naive cells; and 396,288 from cell line to Brain hippocampus middle. The lengths of the sequences vary from 200 to 2,000 nucleotides.

For each sequence, we calculate 14 average energy, and, respectively, 14 lexicon complexity values corresponding to the patterns of lengths between 3 and 16 


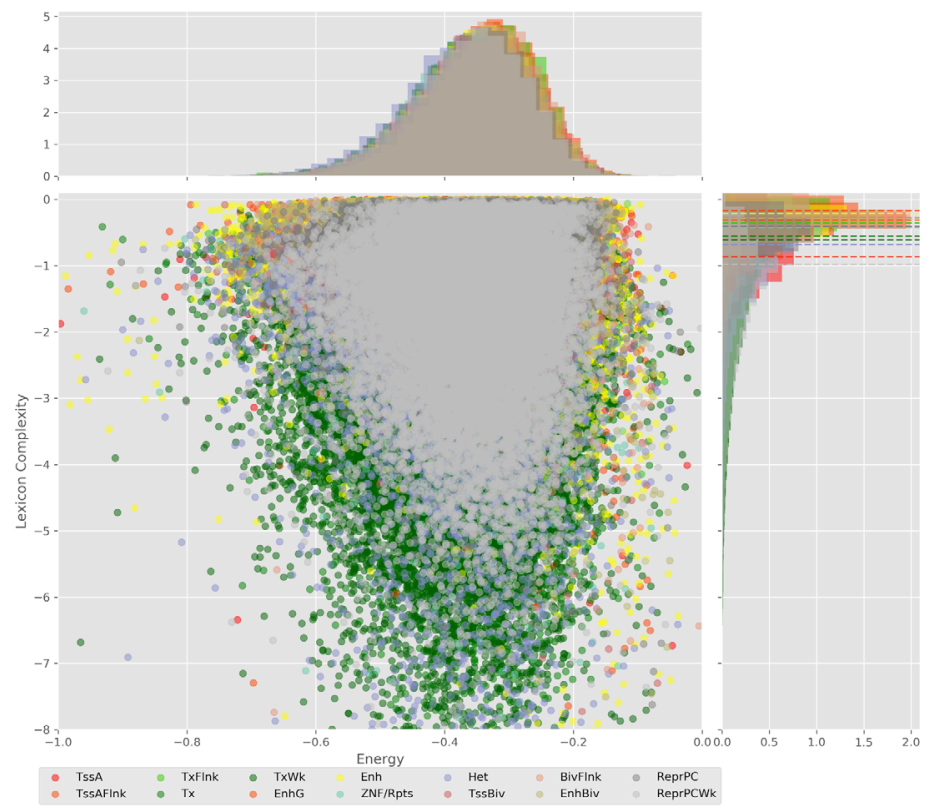

FIGURE 4. Energy against the 6-lexicon complexity (lower left) of the DNA sequences for he 14 regulatory regions of cell line $\mathrm{H} 1$ cells. The histograms correspond to the distribution of the energy (upper) and the distribution of the lexicon complexity (right). The dotted lines represent the most common value of the respective regulatory region.

nucleotides. The average energy and the lexicon complexity are indicators that point out to the potential capacity, and respectively, information encoding quality/degeneracy in DNA sequences ${ }^{[26-30]}$.

Figure 4 displays the potential capacity against quality dynamics for the regulatory sequences of the cell line $\mathrm{H} 1$ cells and their $\mathrm{k}$-lexicons with $k=6$. The histograms of the energy (upper left, Figure 4) can be interpreted the way that the potential to encode information at the length of 6 nucleotides tends to be similar in the sequences of any regulatory region. The same behaviour can be observed in the rest of the lengths (see figures S1-S13) that likely indicates that there is no difference in codifying information for any word length.

However, the lexicon complexity for $k=6$ (right, Figure 4) behaves in a different way for different regulatory regions. The dotted lines represent the common value of lexicon complexity in the sequences of the 14 regulatory regions. This feature suggests that there is a difference, at least for this length, between the regulatory regions in the information encoding quality. Let us note that biological information encoding quality in DNA sequences could be interpreted as a degree of degeneracy.

Degeneracy is a biological phenomenon that means the ability of elements that are structurally dissimilar to perform the same function or yield the same output

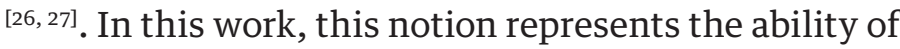
multiple sequences with a certain potential capacity to codify a unique biological function, a regulatory function.

The different degeneracy values in the regulatory regions indicate that the diversity of the words in their respective lexicons is different depending on the region, and meaning, as well as the numbers of words (signs) that encode such regulatory function (significant). As for their codes, i. e. the relationships between corresponding sets of signs and significant, they may 

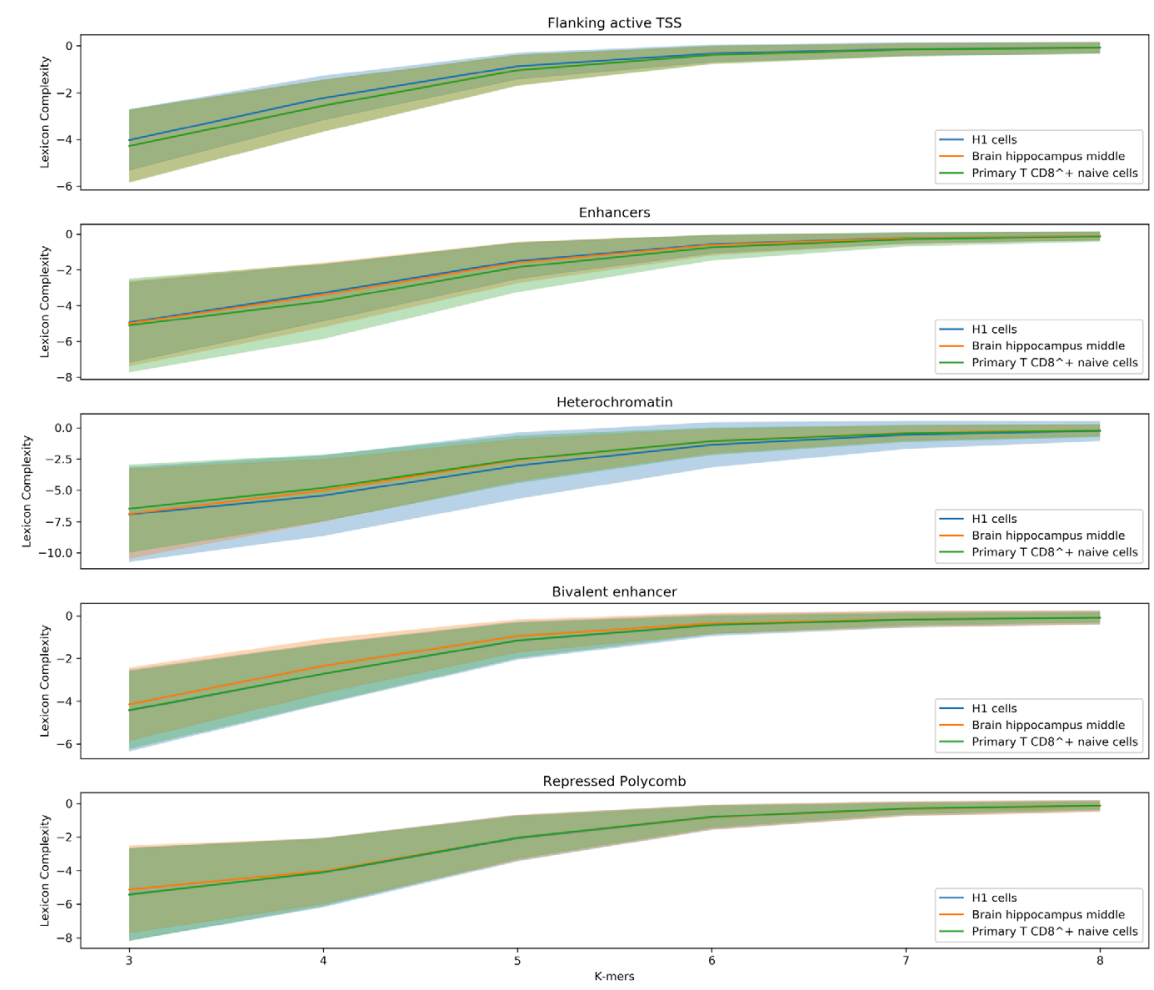

FIGURE 5. Distribution of the lexicon complexity for the regulatory regions Flanking active TSS,

Enhancer, Heterochromatin, Bivalent enhancer and Repressed Polycomb in the cell lines $\mathrm{H} 1$ cells,

Brain hippocampus middle, and Primary T CD8+ naive cells. The bold line represents the mean value of the lexicon complexity of the sequence in the respective cell line for each regulatory region, while the shadow areas represent the standard deviation of the lexicon complexities values.

be different, too. Nevertheless, in this work we don't explore the specific words for each of the single regulatory function lexicons, and, thus we describe the code of each regulatory region as a whole.

The similar behavior, i. e. different degeneracy values in different regulatory regions, can be observed for $k=4,5$, and 7 nucleotides, meanwhile, for the other lengths, the degree of degeneracy tends to be similar for all the regulatory regions. This interesting fact can be explained in the following way: a small word length (1, 2, and 3 nucleotides) enables a brief lexicon and the number of regulatory regions coded would be correspondingly small, while a bigger word length (above 7), leads to a wider vocabulary, and, thus, to an enormous amount of energy needed to maintain the code. On the one hand, it contradicts the less effort principle of nature; on the other hand, a very specific code obtained with the number of signs approximately equal to the number of significances would be easier to "hack" what is a risk for the robustness of an organism.

Although the evidence of different degrees of degeneracy refers to the feasibility of a code for the regulatory sequences, it does not yet indicate that the sequence itself plays a role in the context of the regulatory machinery that determines the cell lines.

In order to explore the influence of the sequence's composition, we are comparing lexicon complexities of the five regulatory regions (Flanking active TSS, Enhancer, Heterochromatin, Bivalent enhancer, and Repressed Polycomb) that the Epigenomic Roadmap uses to propose the lineage of the cell lines. 

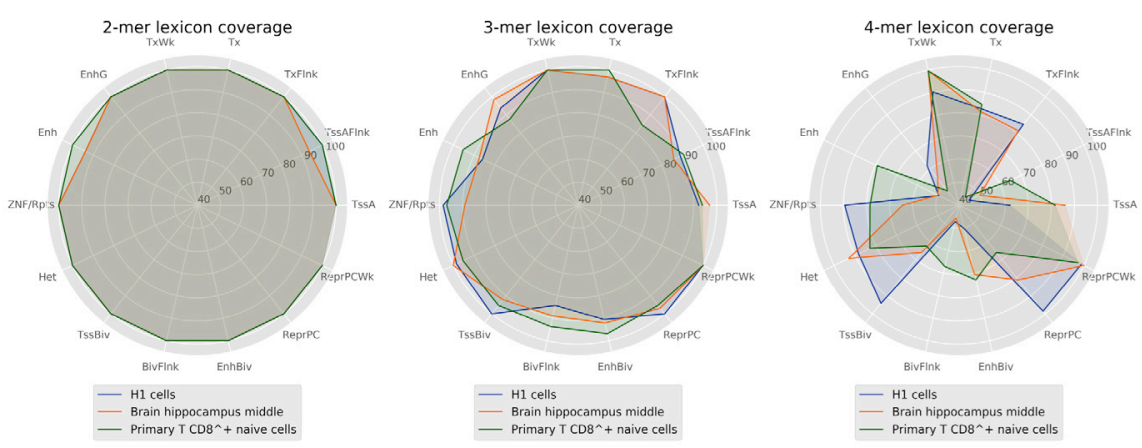

FIGURE 6. Percentage of words covered by the lexicons between 2 and 4 lengths by regulatory regions.

Figure 5 shows value distributions from 3 to 8 lexicon complexities for the three cell lines used in this work. These results present differences between the degree of degeneracy of the regulatory regions of the different cell lines. This is an interesting finding hinting that the sequences could act to establish the regulatory machinery that determines a cell line.

Moreover, another notable result is the decreasing deviation of the lexicon complexity when the length of the studied words increases. As stated earlier, a larger length of words enables producing very specific words, meanings, leading to a specialized lexicon. In this context, it is natural thinking that two sequences have to be similar in their biological functionality when sharing a highly specific word.

However, it may be a coincidence and the vocabulary may still not be sufficiently robust for encoding a biological function. Otherwise, in the case of a shorter word length, the generated vocabulary would be narrow and the set of shared words between sequences may contain the whole vocabulary (Figure 6).

This likely leads to an ambiguous code and a highly probable regulatory function. At the same time, lexicons with medium words lengths (4 to 6 nucleotides) provide enough word diversity/degeneracy degree relationship to support a robust code that may encode the regulatory function sequences and determine a cell line.

\section{CONCLUSION}

The role that non-coding regions plays in DNA sequences is fuzzy due to the diversity and apparent randomness of the sequences. This leads to the notion that these regions are a quiescent part of the genome. However, consortiums as ENCODE, and Epigenomic Roadmap have identified genome regulatory functions in the environment of this part. At the same time, these consortiums do not explore the role of sequences' composition in the determination of the corresponding regulatory function.

Our results show important differences between the lexica of sequences of regulatory regions. While the potential capacity to encode the biological function is similar for any word length, the suitable range of word lengths is between 4 and 7 nucleotides in order of providing sufficient diversity to support the robustness of a code. This is feasible since the degree of degeneracy in these lexica is high enough for the code not to be ambiguous or highly specialized, i. e. the code is robust enough and, hence, not easy to "hack". Furthermore, a broader study could identify the specific words, syntax, and the code that establishes the regulatory function in a sequence, and consequently, determines the cell line to be developed, i. a. taking into account the aspect of the noise immunity of the code ${ }^{[31]}$.

\section{ACKNOWLEDGMENTS}

This work was supported by the grant project CB-256465 CONACyT Basic Science 2015. 


\section{REFERENCES}

[1] Goldman AD, Landweber LF. What is a Genome? Doolittle WF, editor. PLOS Genet [Internet]. 2016 Jul 21;12(7):e1006181. Available from: http://dx.plos.org/10.1371/journal.pgen.1006181

[2] Human Genome Sequencing Consortium I. Finishing the euchromatic sequence of the human genome. Nature [Internet]. 2004 Oct 21;431(7011):931-45. Available from: http://www.nature. com/doifinder/10.1038/nature03001

[3] Dunham I, Kundaje A, Aldred SF, Collins PJ, Davis CA, Doyle F, et al. An integrated encyclopedia of DNA elements in the human genome. Nature [Internet]. 2012 Sep 5;489(7414):57-74. Available from: $h t t p: / / w w w . n a t u r e . c o m / d o i f i n d e r / 10.1038 /$ nature11247

[4] Kundaje A, Meuleman W, Ernst J, Bilenky M, Yen A, HeraviMoussavi A, et al. Integrative analysis of 111 reference human epigenomes. Nature [Internet]. 2015 Feb 19;518(7539):317-30. Available from: http://www.ncbi.nlm.nih.gov/pubmed/25693563

[5] Ernst J, Kheradpour P, Mikkelsen TS, Shoresh N, Ward LD, Epstein $\mathrm{CB}$, et al. Mapping and analysis of chromatin state dynamics in nine human cell types (supplementary). Nature [Internet]. 2011 May 5;473(7345):43-9. Available from: http://www.nature.com/ doifinder/10.1038/nature09906

[6] Strahl BD, Allis CD. The language of covalent histone modifications. Nature [Internet]. 2000 Jan 6;403(6765):41-5. Available from: http://www.nature.com/doifinder/10.1038/47412

[7] Jenuwein T, Allis CD. Translating the histone code. Science (80- )

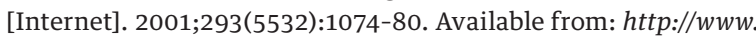
ncbi.nlm.nih.gov/pubmed/11498575

[8] Turner BM. Defining an epigenetic code. Nat Cell Biol [Internet]. 2007;9(1):2-6. Available from: http://www.nature.com/ doifinder/10.1038/ncb0107-2

[9] Allis CD, Jenuwein T. The molecular hallmarks of epigenetic control. Nat Rev Genet [Internet]. 2016 Jun 27;17(8):487-500. Available from: http://dx.doi.org/10.1038/nrg.2016.59

[10] Gardner KE, Allis CD, Strahl BD. Operating on chromatin, a colorful language where context matters. J Mol Biol [Internet].

2011;409(1):36-46. Available from: http://dx.doi.org/10.1016/j. jmb.2011.01.040

[11] Cohanim AB, Kashi Y, Trifonov EN. Three sequence rules for chromatin. J Biomol Struct Dyn [Internet]. 2006;23(5):559-66. Available from: https://www.tandfonline.com/doi/abs/10.1080/073 91102.2006.10507081

[12] Segal E, Fondufe-Mittendorf Y, Chen L, Thåström A, Field Y, Moore IK, et al. A genomic code for nucleosome positioning. Nature

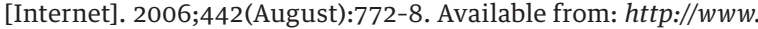
ncbi.nlm.nih.gov/pubmed/16862119

[13] Audit B, Vaillant C, Arnéodo A, D’Aubenton-Carafa Y, Thermes C. Wavelet Analysis of DNA Bending Profiles reveals Structural Constraints on the Evolution of Genomic Sequences. J Biol Phys [Internet]. 2004;30(1):33-81. Available from: http://link.springer. com/10.1023/B: JOBP.0000016438.86794.8e

[14] Salih B, Tripathi V, Trifonov EN. Visible periodicity of strong nucleosome DNA sequences. J Biomol Struct Dyn [Internet]. 2015 Jan 2;33(1):1-9. Available from: http://www.tandfonline.com/doi/ abs/10.1080/07391102.2013.855143?url_ver $=Z 39.88-2003 \&$ rfr id=ori:rid:crossref.org\&rfr_dat=cr_pub\%3Dpubmed
[15] Audit B, Vaillant C, Arneodo A, D'Aubenton-Carafa Y, Thermes C. Long-range Correlations between DNA Bending Sites: Relation to the Structure and Dynamics of Nucleosomes. J Mol Biol [Internet]. 2002 Mar;316(4):903-18. Available from: http://www.ncbi.nlm.nih. gov/pubmed/11884131

[16] Barbieri M. Code Biology [Internet]. Cham: Springer International Publishing; 2015. Available from: http://link.springer. com/10.1007/978-3-319-14535-8

[17] Barbieri M. What is code biology? Biosystems [Internet]. 2018 Feb;164:1-10. Available from: http://dx.doi.org/10.1016/j. biosystems.2017.10.005

[18] Barbieri M. The Code Paradigm. In: Code Biology [Internet]. Cham: Springer International Publishing; 2015. p. 19-34. Available from: https://books.google.de/books/about/Code_Biology. html?id=rNp5BgAAQBAJ\&pgis=1

[19] Hofmeyr J-HS. The first Special Issue on code biology - A bird's-eye view. Biosystems [Internet]. 2018 Feb;164:11-5. Available from: http://dx.doi.org/10.1016/j.biosystems.2017.12.007

[20] Prakash K, Fournier D. Evidence for the implication of the histone code in building the genome structure. Biosystems [Internet]. 2018 Feb;164:49-59. Available from: http://dx.doi.org/10.1016/j.biosystems.2017.11.005

[21] Tsonis AA, Schultz C, Tsonis PA. Zipf's law and the structure and evolution of languages. Complexity [Internet]. 1997 May;2(5):12-3. Available from: http://doi.wiley.com/10.1002/\%28SICI\%291099-0 526\%28199705/06\%292\%3A5\%3C12\%3A\%3AAIDCPLX3\%3E3.0.CO\%3B2-C

[22] Lestrade S. Unzipping Zipf's law. Cai Z, editor. PLoS One [Internet]. 2017 Aug 9;12(8):e0181987. Available from: http://dx.plos. org/10.1371/journal.pone.0181987

[23] Piantadosi ST. Zipf's word frequency law in natural language: A critical review and future directions. Psychon Bull Rev. 2014;21(5):1112-30.

[24] Balasubrahmanyan VK, Naranan S. Information Theory and Algorithmic Complexity: Applications to Language Discourses and DNA Sequences as Complex Systems Part II: Complexity of DNa Sequences, Analogy with Linguistic Discourses. J Quant Linguist [Internet]. 2000 Aug 1;7(2):153-83. Available from: http://dx.doi. org/10.1076/0929-6174(200008)07:02;1-z;ft153

[25] Ji S. Waves as the Symmetry Principle Underlying Cosmic, Cell, and Human Languages. Information [Internet]. 2017;8(1):24. Available from: $h t t p: / / w w w . m d p i . c o m / 2078-2489 / 8 / 1 / 24$

[26] Whitacre J, Bender A. Degeneracy: A design principle for achieving robustness and evolvability. J Theor Biol [Internet]. 2010;263(1):143-53. Available from: http://dx.doi.org/10.1016/i. itbi.2009.11.008

[27] Mason PH. Degeneracy at Multiple Levels of Complexity. Biol Theory [Internet]. 2010 Sep 14;5(3):277-88. Available from: http:// link.springer.com/10.1162/BIOT_a_00041

[28] Gammerman A, Vovk V. Kolmogorov Complexity: Sources, Theory and Applications. Comput J [Internet]. 1999 Apr 1;42(4):252-5. Available from: http://comjnl.oupjournals.org/cgi/doi/10.1093/comjnl/42.4.252 
[29] Popov O, Segal DM, Trifonov EN. Linguistic complexity of protein sequences as compared to texts of human languages. Biosystems [Internet]. 1996 Jan;38(1):65-74. Available from: http://linkinghub. elsevier.com/retrieve/pii/030326479501568X

[30] Orlov YL, Te Boekhorst R, Abnizova II. Statistical Measures of the Structure of Genomic Sequences: Entropy, Complexity, and Position Information. J Bioinform Comput Biol [Internet]. 2006 Apr;04(02):523-36. Available from: http://www.ncbi.nlm.nih.gov/ pubmed/16819800
[31] Fimmel E, Strüngmann L. Mathematical fundamentals for the noise immunity of the genetic code. Biosystems [Internet]. 2018 Feb;164:186-98. Available from: https://doi.org/10.1016/j.biosystems.2017.09.007 


\section{SUPPLEMENTARY FIGURES}

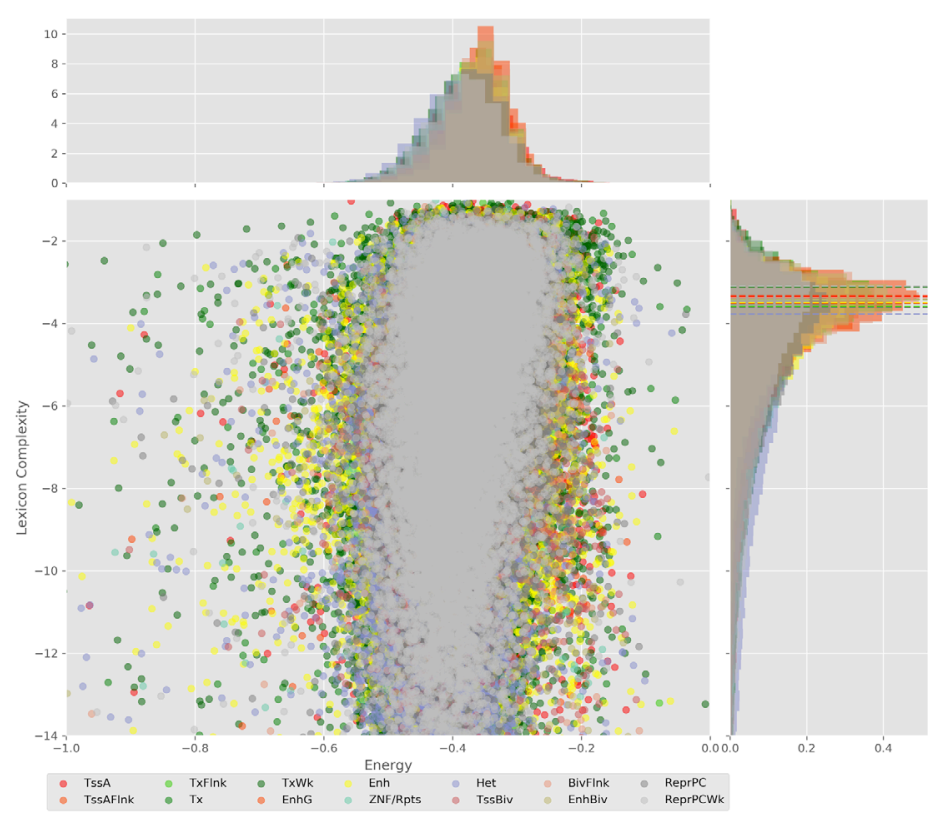

FIGURE S1. Energy against the 3-lexicon complexity (lower left) of the DNA sequences for the 14 regulatory regions of cell line $\mathrm{H} 1$ cells. The histograms correspond to the distribution of the energy (upper) and the distribution of the lexicon complexity (right). The dotted lines represent the most common value of the respective regulatory region.

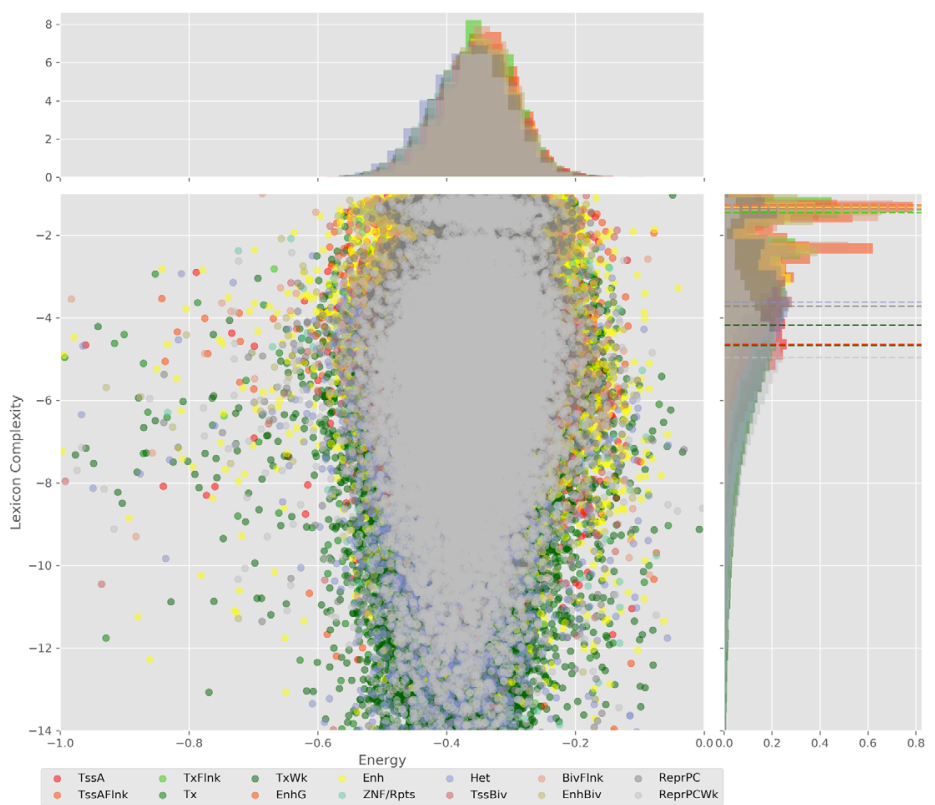

FIGURE S2. Energy against the 4-lexicon complexity (lower left) of the DNA sequences for the 14 regulatory regions of cell line $\mathrm{H} 1$ cells. The histograms correspond to the distribution of the energy (upper) and the distribution of the lexicon complexity (right). The dotted lines represent the most common value of the respective regulatory region. 


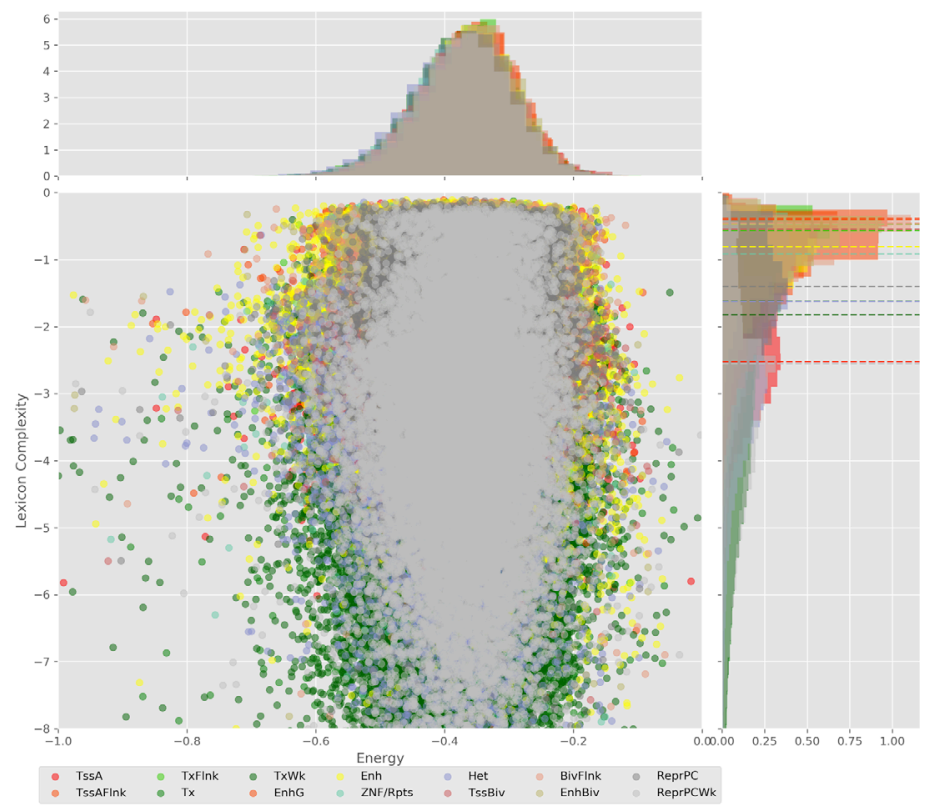

FIGURE S3. Energy against the 5-lexicon complexity (lower left) of the DNA sequences for the 14 regulatory regions of cell line $\mathrm{H} 1$ cells. The histograms correspond to the distribution of the energy (upper) and the distribution of the lexicon complexity (right). The dotted lines represent the most common value of the respective regulatory region.

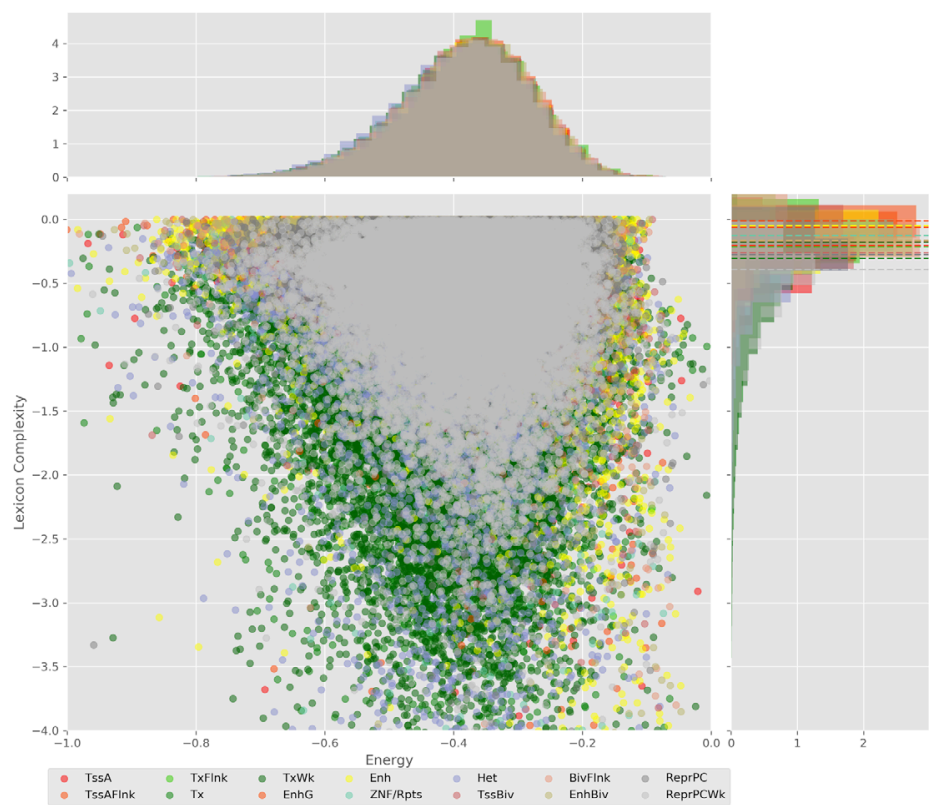

FIGURE S4. Energy against the 7-lexicon complexity (lower left) of the DNA sequences for the 14 regulatory regions of cell line $\mathrm{H} 1$ cells. The histograms correspond to the distribution of the energy (upper) and the distribution of the lexicon complexity (right). The dotted lines represent the most common value of the respective regulatory region. 


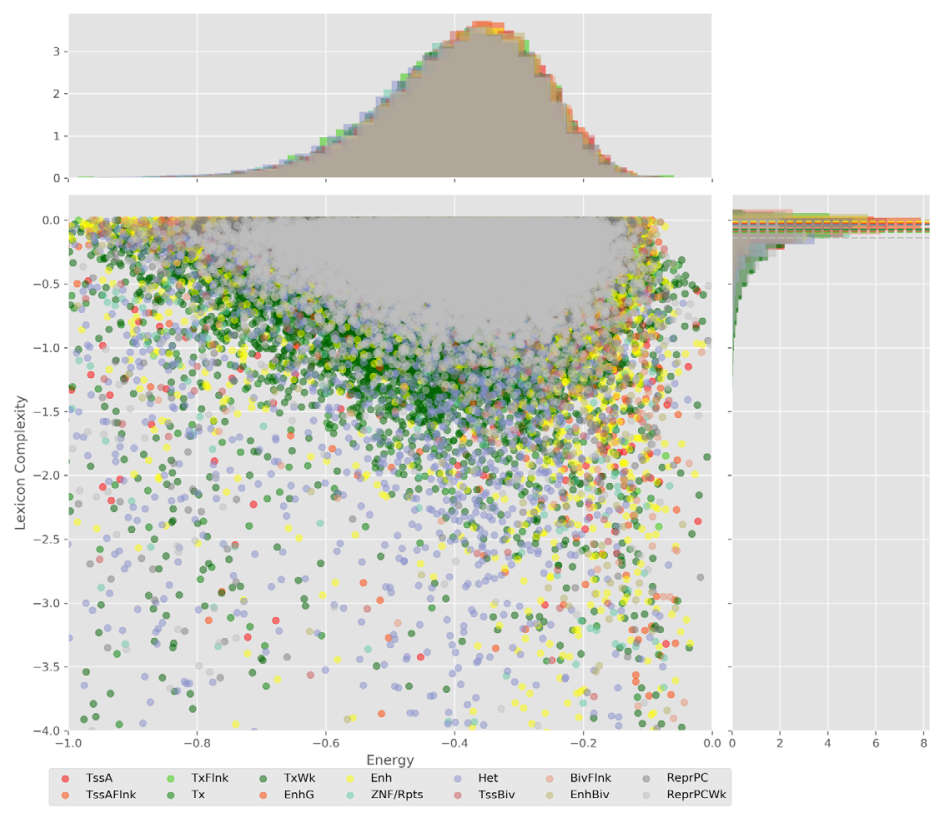

FIGURE S5. Energy against the 8-lexicon complexity (lower left) of the DNA sequences for the 14 regulatory regions of cell line $\mathrm{H} 1$ cells. The histograms correspond to the distribution of the energy (upper) and the distribution of the lexicon complexity (right). The dotted lines represent the most common value of the respective regulatory region.

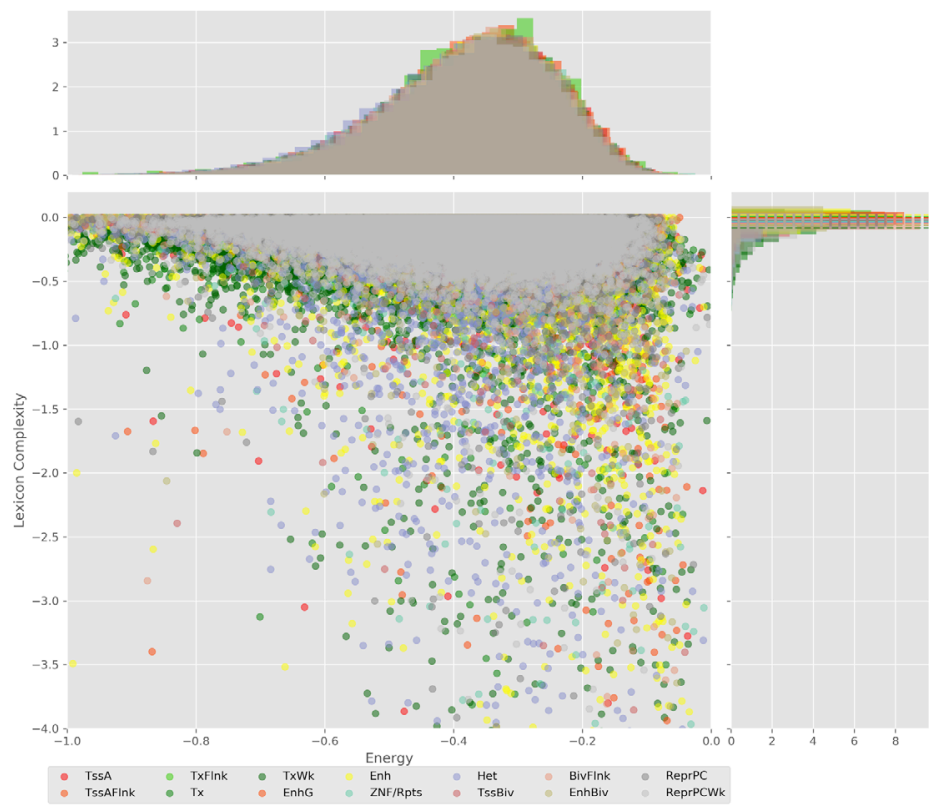

FIGURE S6. Energy against the 9-lexicon complexity (lower left) of the DNA sequences for the 14 regulatory regions of cell line $\mathrm{H} 1$ cells. The histograms correspond to the distribution of the energy (upper) and the distribution of the lexicon complexity (right). The dotted lines represent the most common value of the respective regulatory region. 


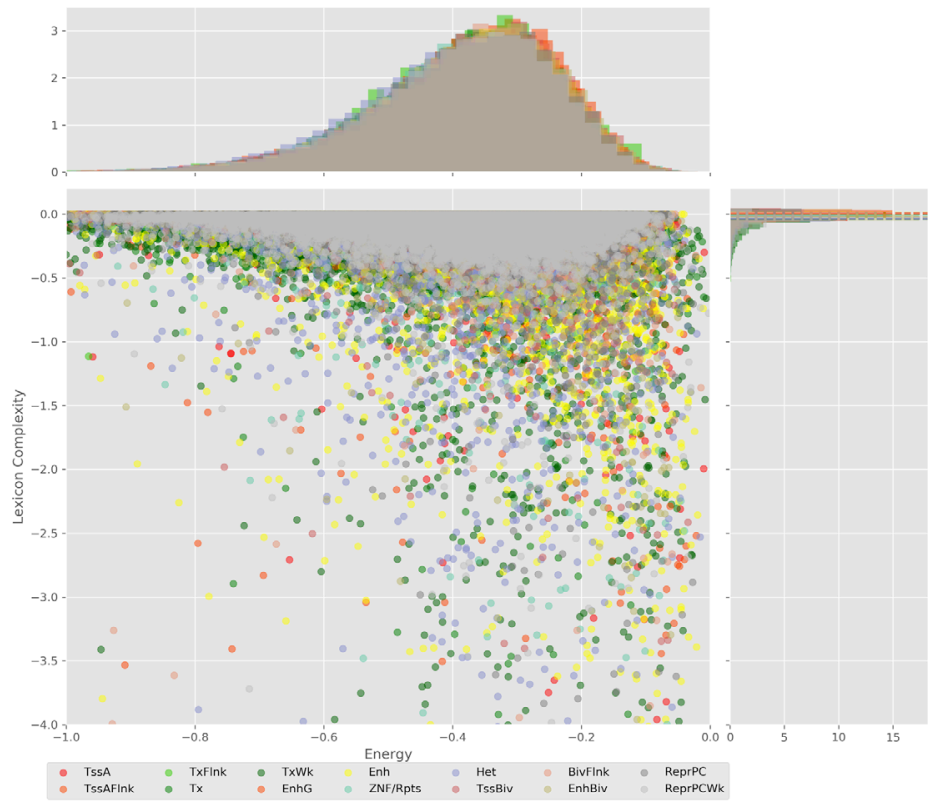

FIGURE S7. Energy against the 10-lexicon complexity (lower left) of the DNA sequences for the 14 regulatory regions of cell line $\mathrm{H} 1$ cells. The histograms correspond to the distribution of the energy (upper) and the distribution of the lexicon complexity (right). The dotted lines represent the most common value of the respective regulatory region.

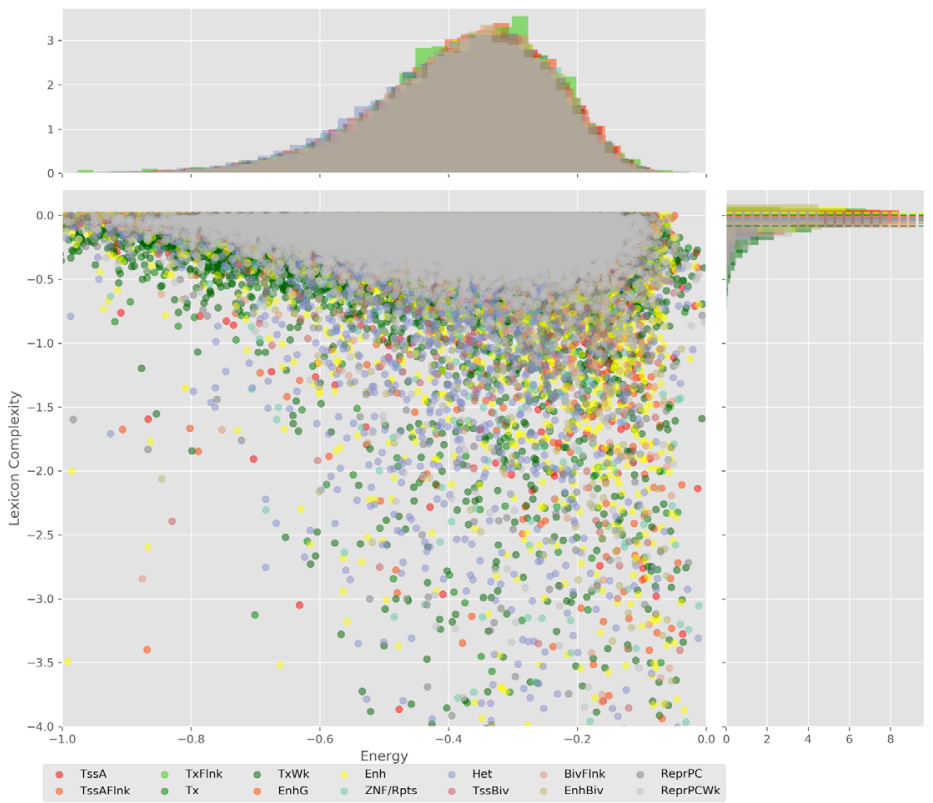

FIGURE S8. Energy against the 11-lexicon complexity (lower left) of the DNA sequences for the 14 regulatory regions of cell line $\mathrm{H} 1$ cells. The histograms correspond to the distribution of the energy (upper) and the distribution of the lexicon complexity (right). The dotted lines represent the most common value of the respective regulatory region. 


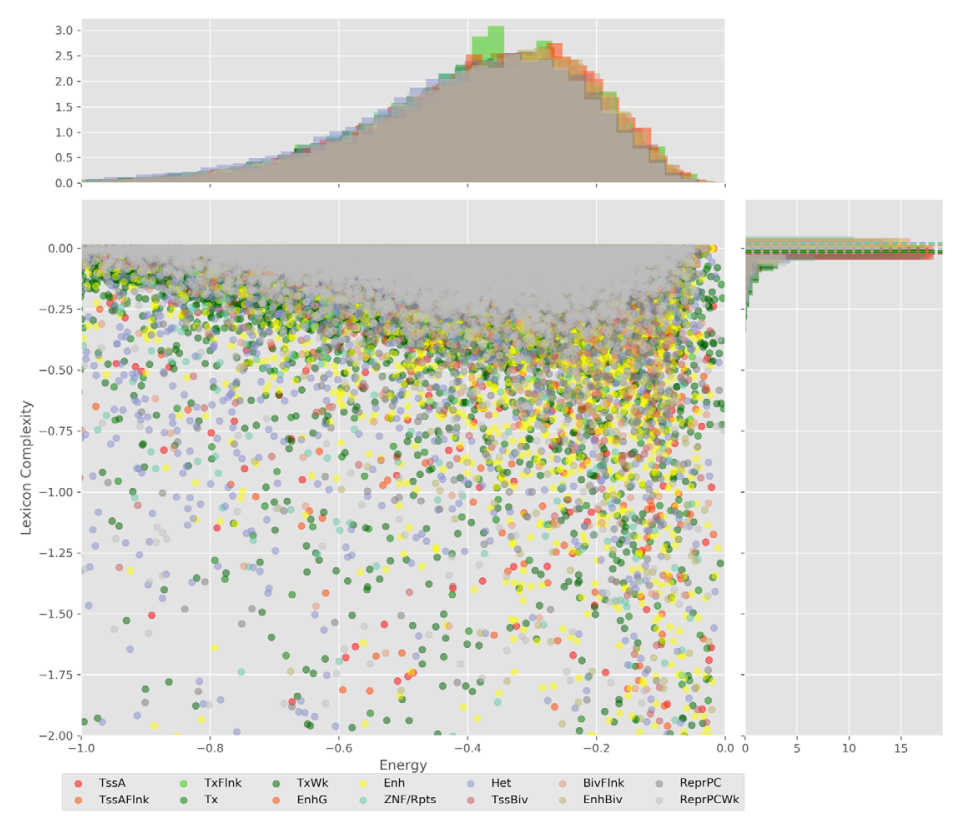

FIGURE S9. Energy against the 12-lexicon complexity (lower left) of the DNA sequences for the 14 regulatory regions of cell line $\mathrm{H} 1$ cells. The histograms correspond to the distribution of the energy (upper) and the distribution of the lexicon complexity (right). The dotted lines represent the most common value of the respective regulatory region.

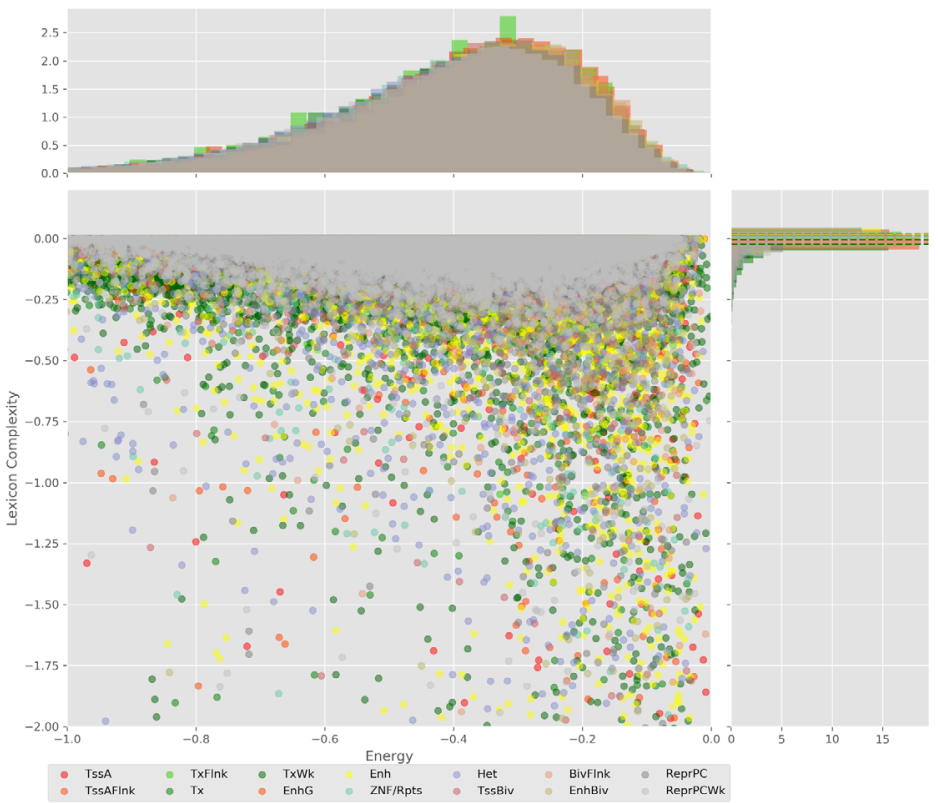

FIGURE S10. Energy against the 13-lexicon complexity (lower left) of the DNA sequences for the 14 regulatory regions of cell line $\mathrm{H} 1$ cells. The histograms correspond to the distribution of the energy (upper) and the distribution of the lexicon complexity (right). The dotted lines represent the most common value of the respective regulatory region. 


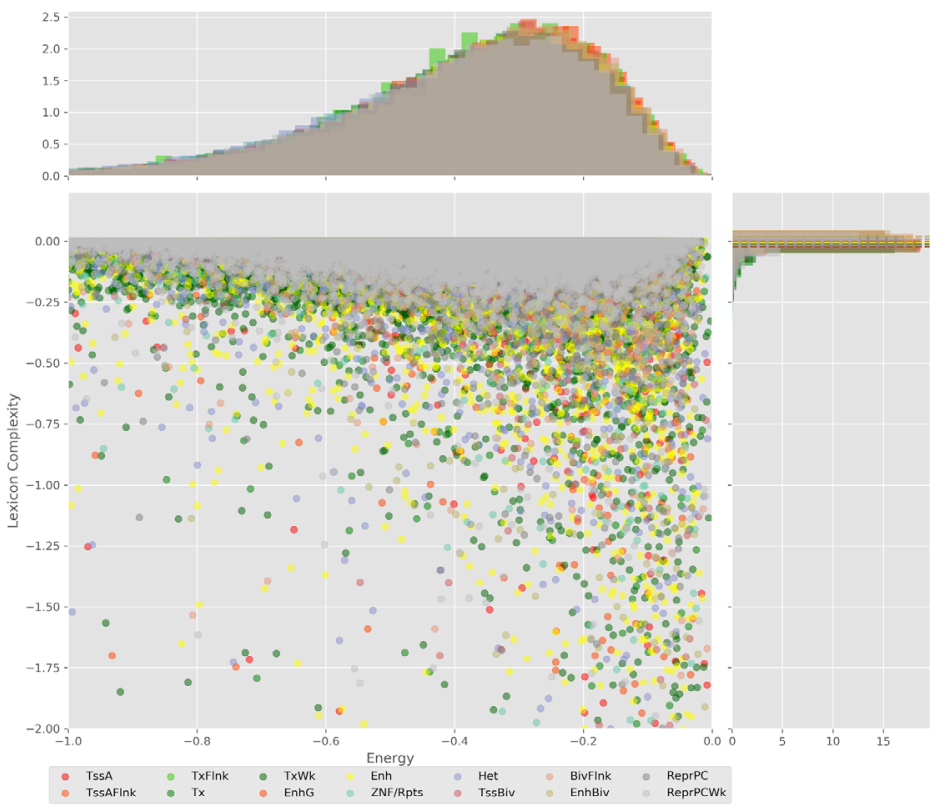

FIGURE S11. Energy against the 14-lexicon complexity (lower left) of the DNA sequences for the 14 regulatory regions of cell line $\mathrm{H} 1$ cells. The histograms correspond to the distribution of the energy (upper) and the distribution of the lexicon complexity (right). The dotted lines represent the most common value of the respective regulatory region.

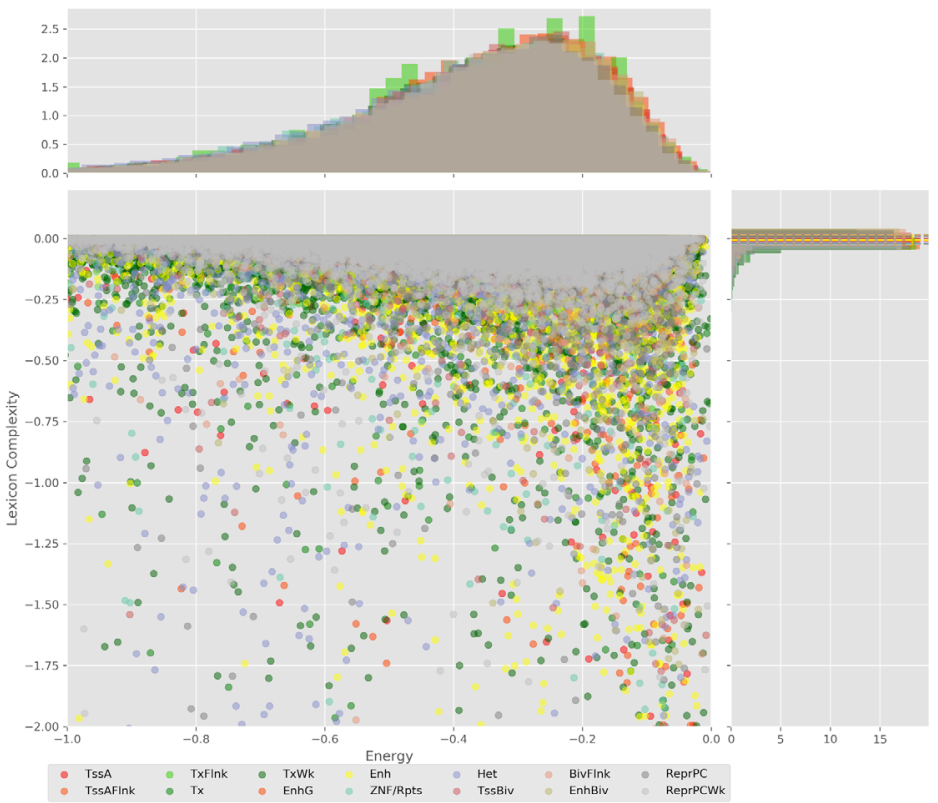

FIGURE S12. Energy against the 15-lexicon complexity (lower left) of the DNA sequences for the 14 regulatory regions of cell line $\mathrm{H} 1$ cells. The histograms correspond to the distribution of the energy (upper) and the distribution of the lexicon complexity (right). The dotted lines represent the most common value of the respective regulatory region. 


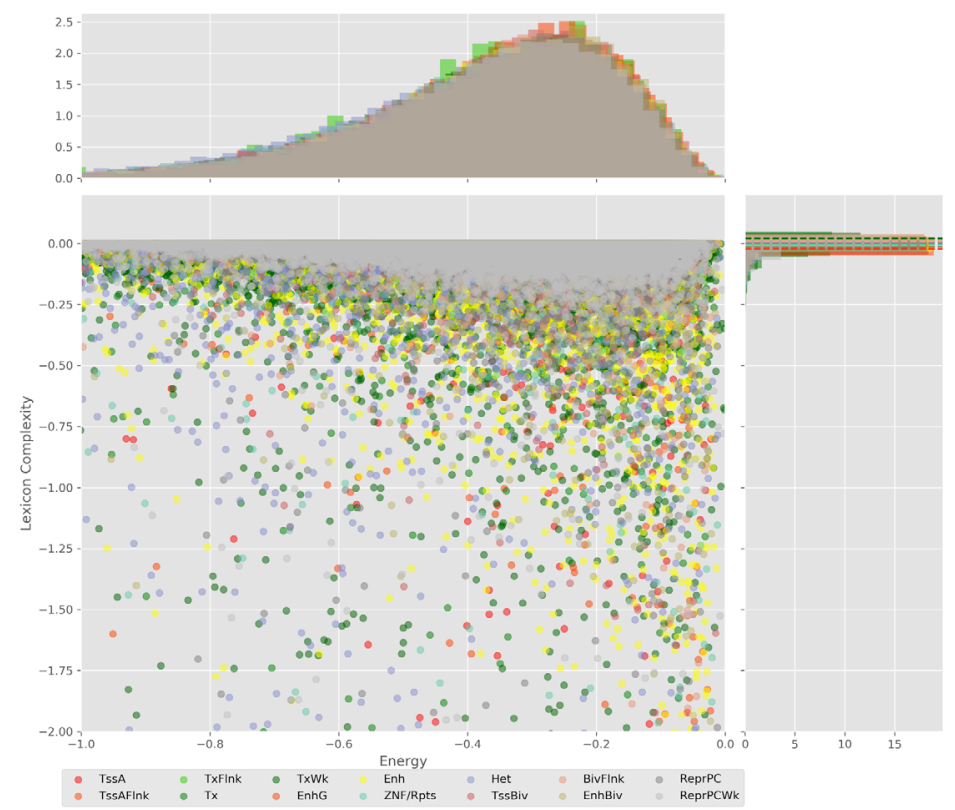

FIGURE S13. Energy against the 16-lexicon complexity (lower left) of the DNA sequences for the 14 regulatory regions of cell line $\mathrm{H} 1$ cells. The histograms correspond to the distribution of the energy (upper) and the distribution of the lexicon complexity (right). The dotted lines represent the most common value of the respective regulatory region. 